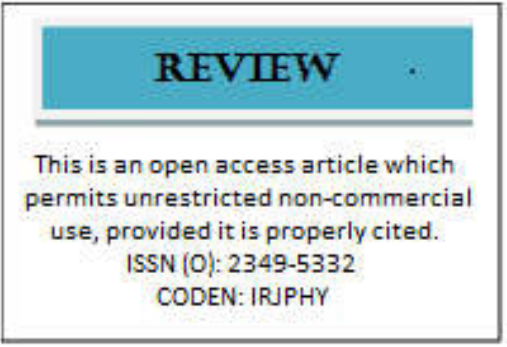

CODEN: IRJPHY
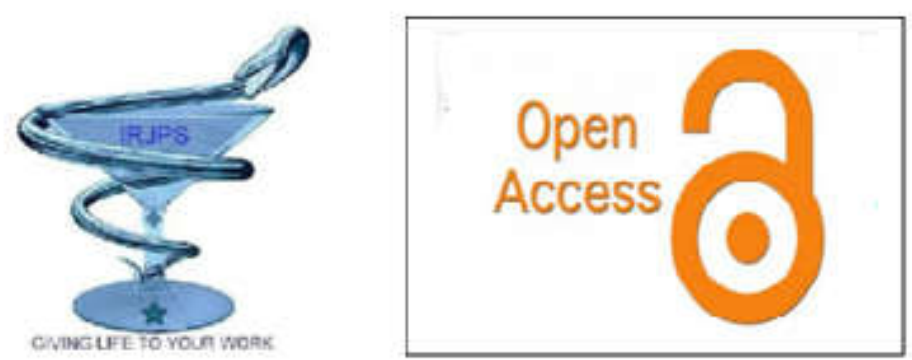

\title{
THE POTENTIAL ADVANTAGES OF SOLID LIPID NANOPARTICLES IN CHEMOTHERAPY OF TUBERCULOSIS
}

\author{
Vipul Sansare*, Abhijeet Kanavaje
}

Department of Pharmaceutics, Indira Institute of Pharmacy, Sadavali, Maharashtra, India.

Submitted on: 17.09.19;

Revised on: 22.09.19;

Accepted on: 28.09.19

\begin{abstract}
Tuberculosis (TB) remains a major global health problem as it is the second leading cause of death after the human immunodeficiency virus (HIV). Conventional chemotherapy fail either because of emergence of multidrugresistance or poor patient compliance to the drug regimen. The aim of this review is to give an update on advantages of solid lipid nanoparticles for treatment of tuberculosis and current antitubercular chemotherapies. Direct lung delivery of anti-TB drugs using pulmonary delivery systems is then reviewed since it appears as an interesting strategy to improve first and second line drugs. A particular focus is place on research performed on inhalable dry powder formulations of antitubercular drugs to target alveolar macrophages where the bacteria develop. Numerous studies show that anti-TB drugs can be incorporated into lipid nanoparticles which can be delivered as dry powders to the deep lungs for instantaneous, targeted and/or controlled release. These new formulations appear as interesting alternatives to deliver directly drugs to the lungs and favor efficient TB treatment.
\end{abstract}

KEYWORDS: Tuberculosis, Solid Lipid Nanoparticles, Oral and inhalable drug delivery, Targeted delivery to alveolar macrophages.

Corresponding author: Vipul Sansare

E mail: avipulsansare@gmail.com

Mobile: +91-8692930764
Indian Research Journal of Pharmacy and Science; 22(2019)1975-1990;

Journal Home Page: https://www.irjps.in DOI: 10.21276/irjps.2019.6.3.8 
INTRODUCTION: Tuberculosis (TB) has caused significant health care problem since long times, particularly in developing countries. $\mathrm{TB}$ is an infectious disease caused by the bacillus Mycobacterium tuberculosis (M.tb), has resulted in more deaths than any other communicable disease. According to World Health Organization (WHO) global TB report 2018, India accounts for about a quarter of the global TB burden. Due to the long treatment period and patient noncompliance, leading frequently to the emergence of multidrug resistant (MDR) strains the chemotherapy for the treatment of TB is extremely difficult. The therapy becomes more complicated and compromised with the emergence of HIV/AIDS pandemic. In this context, new and improved drug delivery strategies for existing drugs may play a crucial role in the $\mathrm{TB}$ management. ${ }^{1}$ Targeted and sustained release chemotherapy offers a great potential in tuberculosis treatment by achieving greater specificity of delivery and improved therapy. ${ }^{2}$ Because M. tuberculosis is known to infect alveolar macrophages (AMs) and affect the pathogenesis of tuberculosis, there have been renewed interests in targeting of antituberculosis drugs to these cells. ${ }^{3,4}$

As per current therapy point of view, combination of antibiotics administered either by oral or parenteral route. The oral route is convenient and safe. However, slower onset of action, first pass metabolism and dose related side effects limits the activity of orally administered drugs. The parenteral route has the highest bioavailability and drug administered parenterally shows quick onset of action. However unfavorable biodistribution leads to accumulation of drug in off-target tissue and rapid clearance form blood by liver or kidney are major problems. $^{5}$
Conventional administration routes may results in poor pulmonary distribution of drugs leads to difficulty in achievement therapeutics concentration of drug at site of action. Besides these limitations, prolongation of treatment, narrow therapeutic window and patient compliance are critical issues. ${ }^{5}$

Nanocarriers mediated drug delivery is new approach to overcome these limitations. Solid lipid nanoparticles (SLNs) are nanometer sized chemical structure having size range 1-1000 $\mathrm{nm}$. SLNs offer several advantages over conventional drug delivery. For one thing, they protect the encapsulated drugs from rapid degradation and excretion. The drugs associated with nanocarriers gradually dissociate and show slow, sustained drug release, thus reduces the frequency of administration. ${ }^{6}$ Lipids used in fabrication of SLNs are biodegradable, nonimmunogenic; whose functionality can be easily modified and hence enhancing tendency of phagocytic uptake by macrophage cells. ${ }^{7}$

This can provide localization at diseased site thus leads to targeted drug delivery. Targeting can leads to enhances treatment effects as well as reduced offtarget side effects. ${ }^{4}$ Therefore, SLNs represent appealing nanocarriers systems for anti-TB drugs and possess large potential for the treatment of pulmonary TB.

This article focuses on the role of SLNs as nanodelivery system for delivery of anti-TB drugs.

\section{Mycobacterium tuberculosis infection}

TB infection occurs via the inhalation of M.tb bacilli and they are known to utilise alveolar macrophages (AMs) as their niche environment for survival. M.tb is transmitted predominantly through small airborne droplets or droplet nuclei created by the coughing or sneezing of a person with pulmonary or laryngeal TB. The inhaled droplets of $1-5 \mu \mathrm{m}$ aerodynamic 
diameters get deposited in the alveolar region of the lungs. There they are taken up by alveolar macrophages, which supply the M.tb with a niche atmosphere. M.tb has developed several mechanisms of uptake into macrophages, which are shown in Fig. 1. M.tb survival within the macrophage might be dependent on the capability of the pathogen to begin phagocytosis via a receptor mediated pathway without initiating an anti-microbial response. It has been suggested that M.tb may engage complement receptors that permit them to be phagocytosed through phagosome bound vesicles. Mannose receptors have been shown to play a key role for the uptake of virulent M.tb but not the avirulent strains. ${ }^{8}$ M.tb may enter the cells through a variety of mechanisms. Perseverance within the macrophage is attained by arrested phagolysosome fusion and the bacilli reside in the endosome. Internalized M.tb within the macrophages are then exposed to multiple microbicidal mechanisms including reactive oxygen and nitrogen species, acidic $\mathrm{pH}$, lysosomal enzymes, and toxic peptides; The survival mechanisms of TB bacilli are still not clear. The probable mechanisms reported are arrest of phagosome-lysosomes fusion within the macrophage; thereby allowing its survival. $^{9,10}$ Furthermore, the macrophage begins manufacture of proteolytic enzymes and cytokines to degrade M.tb. The release of cytokines therefore catches the attention of $\mathrm{T}$ lymphocytes to the infected location. In a person with intact cell mediated immunity, granulomas will be formed through accumulation of activated $\mathrm{T}$ lymphocytes and macrophages around the M.tb to enclose the infection. These tumour necrosis factor (TNF)dependent granulomas create a microenvironment that confines the replication and dissemination of M.tb. This situation results in latent TB infection, where the infection is inactive and cannot be transmitted. On the other hand, these inactive M.tbcan be reactivated when the host immune system is weakened (e.g. by HIV infection). Nonetheless, in a person with a weak immune system the granuloma formation fails to entrap or hold the bacteria. As a result, the M.tbwill be able to break out and spread to other alveoli and organs that lead to progressive primary TB. ${ }^{11,12}$

\section{Current prevention and therapy strategies}

\section{Anti-TB chemotherapy}

The goals of chemotherapy are to cure patients, to minimize threat of death and to prevent transmission of bacilli to healthy persons. To achieve these goals, anti-TB antibiotic combination to be taken orally every day for at least sixmonths. Current standard treatment for non-resistant TB includes 2 months administration of multi drug combination of first-line drugs to reduce the rapidly dividing bacilli load. In the continuous phase, a combination of two or three drugs is used for at least 4 months to sterilize lesions containing fewer and slow growing bacilli. ${ }^{5}$

At present, there are five first line anti-TB agents: isoniazid (INH), rifampicin (RIF), pyrazinamide (PZA), ethambutol (ETB) and streptomycin $(\mathrm{SM}) .{ }^{13}$ Recognized second-line drugs include ethionamide $(\mathrm{ETH})$ or prothionamide, kanamycin (KM), cycloserine (CYC), capreomycin (CAP), paraamino-salicylic acid an viomycin. ${ }^{14}$ Structure, target and mechanism of action of current anti-TB drugs are summarized in Table 1.

\section{Adaptive mechanisms developed by M.tb to survive in $\mathbf{A M}$}

M.tb resides in phagosomes and phagolysosomes of AMs. AMs are cells of the monocyte-macrophage system (MMS) which play a key role in innate and acquired immune system (ingesting and killing 
microorganisms). Despite these functional characteristics, macrophages are the target of pathogens (e.g. M. tb) which are well adapted to block anti-infectious immune mechanisms of macrophages. These pathogens are able to survive, to multiply in macrophages. M.tb interferes with the transformation of the primary endosomes, respectively with phagosome maturation (lysosomes fusion and phagosome acidification). ${ }^{15,16}$ Thus fusion of endosomes with lysosome is delayed or blocked.

Pathogen from this group prevents formation of phagolysosome by simultaneous action of several mechanisms. Most common mechanisms are reduction in level of proton ATPase inside the endosomes, inhibition of

nitric oxide synthase and active elimination of Phosphatidylinositol 3 -phosphate (PI3P). ${ }^{17,18}$ To formulate appropriate drug delivery system it is essential to know cellular pharmacokinetics of antibiotics and intracellular niche for survival of the organism.

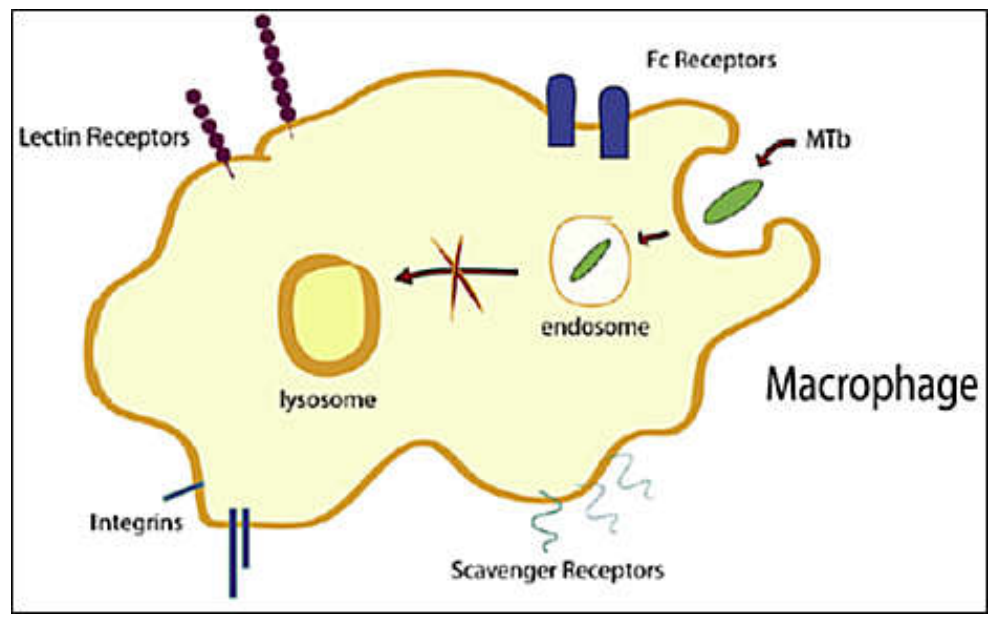

Fig.1: Cell entry and trafficking of $M . t b$ within the macrophage

\section{Targeting of drug delivery systems (DDS) to} alveolar macrophages

Improvement of anti-TB drugs has gained a lot of attention in the past decade. New approaches for TB treatment are becoming essential to combat this disease, especially for patients with co-infection and drug resistance. It is important to target TB-targeting DDS not only to specific cell types but to the correct sub-cellular compartment Mechanism of cellular entry of DDS in alveolar macrophages depends on physicochemical properties of DDS. Various ways are utilized to specifically accumulate DDS in alveolar macrophages. Microparticles enter the cell through phagocytosis and finally release in the cell by lysosomal escape. Liposomes, polymer-based nanoparticles and solid lipid nanoparticles have been explored for $M$. $T b$ therapy and prophylaxis are often coated with specific ligands for macrophage receptors to enhance targeting. Fig. 2 highlights the mechanism of uptake nanosystems in alveolar macrophages. Successful targeted inhalable drug delivery system is one that activates macrophages to initiate phagosome lysosome fusion or facilitate accumulation of drug in phagosome. ${ }^{4}$ 
Table 1: Structures, minimum inhibitory concentration and mechanism of action of anti-TB drugs

\begin{tabular}{|c|c|c|c|}
\hline Drug & structure & MIC & Mechanism of action/target ${ }^{5}$ \\
\hline Isoniazid & & $0.01-0.2$ & $\begin{array}{l}\text { Inhibit mycolic acid synthesis by binding to the NADH- } \\
\text { dependent enoyl acyl carrier protein reductase InhA. } \\
\text { Affects DNA, carbohydrates, lipids and NAD metabolism }\end{array}$ \\
\hline Rifampicin & & $0.05-0.5$ & $\begin{array}{l}\text { Bind to bacterial DNA dependent RNA polymerase and } \\
\text { inhibit DNA transcription in bacilli. }\end{array}$ \\
\hline Pyrazinamide & & $20-100$ & $\begin{array}{l}\text { Disrupt membrane potential and interfere energy } \\
\text { production necessary for survival of bacilli. }\end{array}$ \\
\hline Ethambutol & & $1-5$ & $\begin{array}{l}\text { Bactericidal/ bacteriostatic activity } \\
\text { Inhibit cell wall arabinogalactan biosynthesis }\end{array}$ \\
\hline Streptomycin & & $2-8$ & $\begin{array}{l}\text { Inhibit polypeptide synthesis (translation) by binding to } \\
\text { 30S ribosomal subunit. }\end{array}$ \\
\hline Ethionamide & & $0.6-2.5$ & $\begin{array}{l}\text { Inhibit mycolic acid synthesis by binding to the ACP } \\
\text { reductase InhA. }\end{array}$ \\
\hline Kanamycin & & $1-8$ & $\begin{array}{l}\text { Inhibit polypeptide synthesis (translation) by binding to } \\
\text { 30S ribosomal subunit. }\end{array}$ \\
\hline Cycloserin & & $5-20$ & $\begin{array}{l}\text { Inhibit polypeptide synthesis by inhibiting the d-alanine } \\
\text { racemase enzyme (AlrA). }\end{array}$ \\
\hline Capreomycin & & $5-20$ & $\begin{array}{l}\text { Modify ribosomal structure at the } 16 \mathrm{~S} \text { rRNA and disrupt } \\
\text { protein synthesis. }\end{array}$ \\
\hline & & & $\begin{array}{l}\text { Anionic Microparticles } \\
\quad . \dot{0} \text {. }\end{array}$ \\
\hline
\end{tabular}

Fig.2: Mechanism of uptake of nanosystems in alveolar macrophages 
Passive or active targeting can be achieved with nanoparticulate drug delivery system. Passive targeting is based on difference between normal and pathological cells like overexpression of certain cellular components or surface receptor in infected cell. Small size and longer circulation time of particles, locally increased capillary permeability (EPR effect) facilitate passive entry of nanocarriers at pathological site. With active targeting, the surface of nanoparticle is modified with targeting molecules that interact with their ligands in the tissue and may provide a way for the drugs delivery system to reach the site of interest. ${ }^{4}$
AM receptors for cellular entry as potential target for anti-TB therapy

Large numbers of phagocytosis receptors located on the surface of the AM. These receptors reflect the physiological functions of $\mathrm{AM}$ and are also involve in internalization of $M . T b$ in AM.

M.tbbacilli get enter the alveolar macrophages through various receptors. Accumulation of drug in the same intracellular niches as those in which the microorganism develop could be achieved by therapeutic targeting of receptors. Following are some important receptors present on alveolar macrophages that exploited for targeting of antimicrobial drugs (Fig. 3).

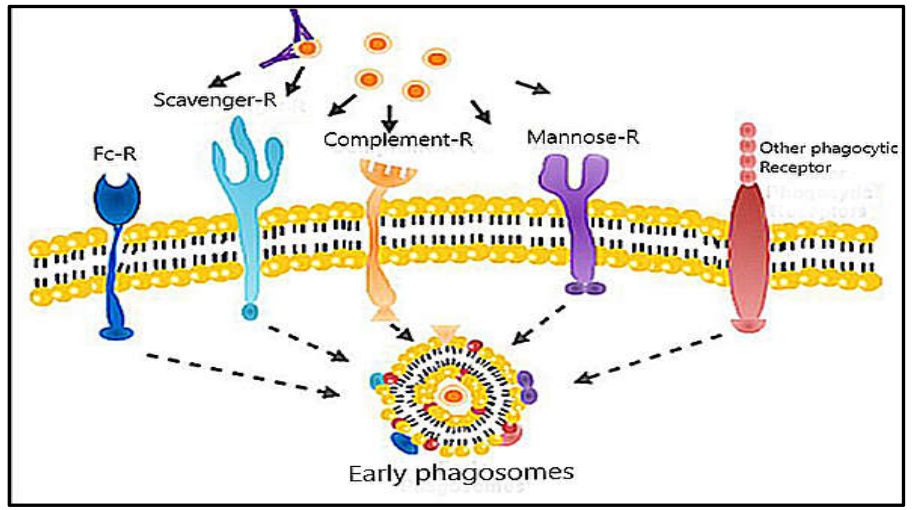

Fig.3: Receptors for cellular entry of $M . t b$ in alveolar macrophages

Fc Receptors: Fc receptor (Fragment crystallizable) is member of the Ig superfamily of receptors, mainly involve in binding of Fc portion of immunoglobulins and produce an antibody-mediated phagocytosis. ${ }^{19}$ Intense production of proinflammatory mediators such as reactive oxygen and nitrogen species and arachidonic acid derivatives takes place after phagocytosis through Fc receptors. Fc receptor was used by Moghiniet al,. ${ }^{20}$ for targeting of opsonized phospholipid vesicles targeted to macrophages and hepatocytes. Broekhovenet $a l, .^{21}$ have targeted liposomes to dendritic cells through $\mathrm{Fc}$ receptors.
Complement Receptor (CR): CR3 receptor (subtype of $\mathrm{CR}$ receptor) is the predominant receptor used by M.tb to enter monocytic cells. The phagocytosis mediated by CR is not coupled with the production of proinflammatory mediators and not involve in the initialization of an inflammatory response. Takagi et $a l, .{ }^{22}$ observed CR3 mediated phagocytosis for macrophage uptake of oligomannose-coated liposomes.

Mannose Receptor (MN):Highly expressed receptor on macrophages surface and recognizes mannose and fucose from the surfaces of microscopic pathogens. ${ }^{19}$ 
Mannose receptor involved mainly in intracellular transport of M.tb and in antigen presentation. ${ }^{23}$ Mannose receptor recognizes carbohydrate moieties of different pathogen ligand, including Candida albican, Leshmania donovani, Trypanosome cruzi and Mycobacterium species. The ability of mannose receptor to recognize pathogen is also though to facilitate infection by $M . t b .^{24}$ These bacteria enters the macrophages through mannose receptor and able to multiply and survive in macrophages. Thus mannose receptor is possible target to specifically accumulate nanocarriers inside the alveolar macrophages to treat M.tb infection.

TB infection leads to AMs activation which over express mannose (MR-CD 206 and CD 163) receptors. Such activated macrophages can recognize and facilitate internalization of carriers bearing mannosylated carbohydrate molecules. Manose receptors were successfully used by Bharadwaj et $a l,{ }^{25}$ for targeting mannan conjugated liposomes containg anti- tb drug to alveolar macrophages.

Scavenger Receptors (SR): Scavenger receptors (SRA and SR-B or CD36) are a broad group of transmembrane receptors which recognize a variety of structures as LDL, phosphatidyl serine, polyanionic ligands, and negatively charged nanoparticles. $^{26}$

\section{Physicochemical aspects of macrophages targeting}

Physicochemical properties of nano-carrier affect its uptake by the alveolar macropgages. Following are some important physicochemical aspects that affect the uptake of nanocarrier.

\section{a. Surface charge}

Surface charge plays crucial role for interaction of particle with macrophages cells and stability. Macrophages cell display negatively charge sialic acid residue on the surface. Thus positively charges particles possess high affinity towards the macrophages cell membrane as compared to that of negatively charges particles. ${ }^{27}$ Similar results were reported by Gallagher et al., ${ }^{28}$ where positively charges particles showed higher binding potential to rat AM through sialic acid as compared to negatively charged particles.

\section{b. Particles size}

Particle size of the particles influences particle macrophages interaction. The particles ranging between 1 to $6 \mu \mathrm{m}$ are most accessible to AM, whereas particle more than $10 \mu \mathrm{m}$ and less than $0.2 \mu \mathrm{m}$ are able to escape phagocytosis. AM mostly engulf nanoparticles through pinocytosis. Similar results were reported by Makino et al., ${ }^{29}$ where polystyrene microsphere having particle size $1 \mu \mathrm{m}$ exhibit higher uptake by AM as compared to that having particle size $6-10 \mu \mathrm{m}$ and $0.2-0.5 \mu \mathrm{m}$.

\section{c. Surface hydrophilicity/ hydrophobicity}

The hydrophilicity or hydrophobicity of carrier materials plays a pivotal role in macrophages targeting. Studies revealed that hydrophobic domains are more preferable for macrophages targeting. Carrier particles with hydrophobic domains are recognized by blood proteins which facilitate its opsonization and phagocytosis by AM. However hydrophilic carrier systems or PEGylated carrier systems, show prolonged circulation time before being recognized by macrophages. According to Fontana et al., ${ }^{30}$ uptake of amoxicillin nanoparticles in to J774 macrophages was significantly reduced when coated with PEG.

\section{Solid lipid nanoparticles}

Background, definition, method of preparation, advantages and disadvantages

Parenteral fat emulsion was the first dosage form developed in 1960s for incorporation of lipophilic 
drugs in lipid droplets. ${ }^{31}$ Miller et al., ${ }^{32}$ was developed the first generation lipid nanoparticles, called SLNs. SLNs are composed of solid lipid dispersed in an aqueous solution of surfactant as stabilizer. Biocompatibility of SLNs can be achieved by using biocompatible and biodegradable lipids and surfactants. Table 2 summarizes lipids and surfactants, which were already used for the preparation of lipid nanoparticles. The mean diameter of SLN ranges from 40 to $1000 \mathrm{~nm}$. SLN provide following features: physical stability, low toxicity, controlled release and protection of encapsulated drug from degradation, if well tolerated excipients are used. Moreover, SLNs can be prepare by organic solvent free technique and can be easily scaled up, e.g., by high pressure homogenization, melt homogenization ultrasonication. Potential disadvantages of SLN are expulsion of drug during storage and low drug loading capacity.

Table 2: Overview of solid lipids, liquid lipids and surfactants which were used to prepare SLN

\begin{tabular}{|c|c|c|}
\hline Class & Examples & References \\
\hline Solid lipids & $\begin{array}{l}\text { Stearic acid } \\
\text { Tristearin } \\
\text { Tripalmitin } \\
\text { Palmitic acid } \\
\text { Precirol ATO } 5 \\
\text { Glycerol monostearate } \\
\text { Compritol } 888 \text { ATO } \\
\text { Cetyl palmitate } \\
\text { Glyceryl palmitostearate }\end{array}$ & $\begin{array}{l}53,38,54 \\
55 \\
55 \\
53 \\
46 \\
56,57 \\
58 \\
59 \\
60\end{array}$ \\
\hline Surfactants & $\begin{array}{l}\text { Poloxamer } 188 \\
\text { Polysorbate } 20 \\
\text { Polysorbate } 80 \\
\text { Polyvinyl alcohol } \\
\text { Soya lecithin } \\
\text { Sorbitan trioleate } \\
\text { Soya phosphatidylcholine } \\
\text { Sodium cholate } \\
\text { Sodium glycocholate }\end{array}$ & $\begin{array}{l}57 \\
46 \\
59 \\
38 \\
55 \\
59 \\
57 \\
57 \\
55\end{array}$ \\
\hline
\end{tabular}

\section{Pulmonary delivery of anti-tb therapeutics}

Novel inhaled anti-TB antibiotics, have led to innovations in designing suitable delivery systems. These emerging design technologies are in urgent demand to certify high aerosolisation performance, reliable efficacy and reasonable patient adherence. Sacks et al. ${ }^{33}$ used aminoglycosides solution (streptomycin, kanamycin, amikacin) administered via nebulization for patients with smear-positive pulmonary TB. As a result, aminoglycosides 
inhibited bacillary growth in alveoli and prevented transmission, but they were not able to affect bacteria inside the macrophages. ${ }^{34}$ Thus it appears that merely aerosolizing an anti-TB drug may not be sufficient. For efficient bacteria killing, drugs need to be formulated into suitable delivery systems ensuring their rapid uptake into macrophages which harbor the TB bacilli.

Many existing anti-TB drugs were considered and suitably formulated for direct delivery to the lungs. To benefit from lung delivery advantages as well as to overcome some challenges encountered in TB treatment, anti-TB drugs were developed with particulate drug delivery systems in the form of liposomes, microparticles and nanoparticles for pulmonary administration. These inhalable nanocarriers have the potential for inhaledanti-TB therapy. They showed lower drug doses, lower toxicity as well as a lower dosing frequency which were beneficial to patients with pulmonary TB for prolonged treatment.

Previous research on inhalable anti-TB drugs focused on preparing liquid formulations administered to the lungs by nebulization. However, liquid formulations as they are typically unstable because of the potential for sedimentation, crystal growth and polymorphism. Therefore, powder formulations seem to be alternative suitable forms for direct delivery of drugs to the lungs. Recently SLNs based dry powder inhalers (DPIs) have become highly favorable for delivering high-dose and single-dose anti-TB drugs with the aid of advanced particle engineering. ${ }^{35}$

\section{SLNs based dry powder inhaler (DPI)}

SLNs for pulmonary drug delivery were formulated with solid lipids and SLNs based dry powders were prepared by following two steps processes. First, drug was entrapped in SLNs matrix by conventional methods. These SLNs dispersion was then dispersed into the carrier (lactose, glucose, trihalose) and mixture was converted into dry powder form by freeze drying, spray drying, supercritical fluid technology. ${ }^{35,36}$ Some studies reported use of Lleucine as a excipient to improve aerosolisation efficiency of dry powder. ${ }^{37}$

SLNs based dry powders offer the advantages such as: direct delivery of the drug within the lungs, possibility of dose reduction, high drug loading, controlled drug release over a prolonged time period, stability and better mucosal adhesion. ${ }^{38}$ Upon inhalation, carrier dissolves in lung fluid and then drug release for a prolonged time. SLN is feasible system for targeting the lung and they can be phagocytosed by immune cells (e.g., macrophages). Moreover, SLNs were efficiently taken up by AMs where TB bacilli reside and therefore can provide a way of targeting anti-TB therapeutics and other agents to immune cells. Table 3 represents the recent studies of SLNs based DPI containing anti-TB drugs.

Table 3: Overview of SLNs based DPI of anti-TB drug

\begin{tabular}{|l|l|l|}
\hline Drug & Method of preparation & References \\
\hline Rifampicin & Ultrasonication, lyophilization & Vieira $^{40}$ \\
\hline Rifampicin & Lipid film hydration, lyophilization & Chuan $^{39}$ \\
\hline Rifampicin & Melt homogenization ultrasonication lyophilization & Maretti $^{41}$ \\
\hline
\end{tabular}


In 2013, Chuanet al., ${ }^{39}$ encapsulated RIF into SLNs for delivery to the respiratory tract. RIF-loaded SLNs dispersion were obtained from mixture of soya lecithin, steric acid and palmitic acid using lipid film hydration and were freeze-dried with maltose as a cryoprotectant. Cytotoxicity evaluation of RIF loaded SLNs on cultured AMs (NR8383) and alveolar epithelial cells (AECs) type II (A549) revealed more than $80 \%$ cell viability which indicated low toxicity to both AMs and AECs. To assess cellular uptake of Fluorescein isothiocynate labelled RIF SLNs, confocal Laser Scanning Microscopy was performed. It was observed that RIF-SLNs delivered markedly higher RFP into AMs than that into AECs. After pulmonary administration of RIF-SLNs in SpragueDawley rats, the amount of RIF in AMs was significantly higher than that in AECs at each time point. Results demonstrated that SLNs are a promising strategy for the delivery of rifampicin to alveolar macrophages selectively

Recently, Vieira et al., $2017^{40}$ have successfully prepared RIF loaded mannosylated SLNs (RIF MSLNs) for targeted drug delivery to alveolar macrophages. RIF was encapsulated inside the solid lipid matrix using hot ultrasonication method. The Dmannose was surface conjugated on preformed SLNs by incubation of SLNs with

mannose in sodium acetate buffer. Characterization of SLNs was carried out with respect to particle size, zeta potential, encapsulation efficiency, transmission electron microscopy (TEM), in-vitro release study, cytotoxicity and cellular internalization study. TEM revealed spherical SLNs with particle size 252nm, positive zeta potential and higher entrapment efficiency. SLNs showed biphasic drug release profile. Cytotoxicity evaluation of RIF M-SLNs and RIF SLNs on Human leukemia monocyte THP 1cells revealed low toxicity to THP 1cells. The in vitro cell uptake of coumarin 6 labelled RIF M-SLNs revealed mannosylation improved the SLNs uptake inside the cells. Results suggested that mannosylated SLNs constitute suitable carriers for the inhalatory administration of RIF, protecting it from the pulmonary fluids and increasing drug's bioavailability.

Marettiet al., $2014^{41}$ formulated RIF loaded inhalable solid lipid microparticles for targeted drug delivery to alveolar macrophages. RIF loaded solid lipid microparticles (SLM) prepared using stearic acid and freeze dried to obtain dry powder. Freeze dried SLMs then characterized for particle size, surface charge, surface morphology, cytotoxicity study, in-vitro release study and in-vitro antibacterial activity against B. subtilis ATCC. Cellular uptake study of prepared SLMs was studied using murine macrophages cell line. The SLMs was found to be suitable for pulmonary drug delivery. In-vitro release study showed sustained release of RIF from SLMs over the period of 96 hours. Cell line study demonstrated nontoxicity of SLMs and ability of uptake inside the alveolar macrophages.

\section{Requirements of SLNs for pulmonary drug delivery}

As mentioned earlier, SLNs offers several advantages for pulmonary application. However SLNs have to meet some demands for pulmonary applications. These requirements are sterility, isotonicity, biocompatibility and $\mathrm{pH}$ value in the neutral range since lungs have only limited buffer capacity.

\section{a)Sterility}

Following sterilization methods are possible for SLNs: autoclaving, radiation sterilization and sterile filtration, if the particle size is less than $200 \mathrm{~nm}^{7}$ During autoclaving, the lipid melts and recrystallizes 
during cooling. This leads to modification in lipid structure, and that could affects drug release behavior. Moreover, on melting lipid droplets may aggregate before reforming lipid nanoparticle leading to larger particles. Therefore, autoclaving results in physical problems, such as aggregation, modulation of release behavior and increase in particle size. Drawback related to gamma radiation is the formation of free radicles which can reacts with active constituent loaded in SLNs or with carrier system. ${ }^{42}$ Successful autoclaving SLNs was mentioned by Cavalli et al., ${ }^{43}$ Heiatiet al., ${ }^{44}$ Nayaket al., ${ }^{45}$ and Pardeike et al., ${ }^{46}$ However it has to be mentioned that, most of the working groups have not focused on chemical stability during sterilization.

\section{b)pH and isotonicity}

SLNs dispersion can be isotonized by adding ionic and nonionic tonicity adjusting agents. Ionic tonicity adjusting agents such as sodium chloride or other salts may affects on stability of SLNs. The addition of electrolytes induces aggregation of SLNs due to reduction in zeta potential. ${ }^{36}$ Nonionic tonicity adjusting agents like glycerol and carbohydrates are preferred to adjust tonicity of SLNs dispersion. If necessary, the $\mathrm{pH}$ of SLNs dispersion can be adjusted to neutral by addition of acids, bases or buffers.

\section{c) Biocompatibility}

Tolerability of SLNs dispersion can be achieved by using biocompatible and biodegradable lipids and stabilizer. It is advisable to use non-toxic surfactants for pulmonary administration. Most of the surfactants showed toxicity after pulmonary administration thus selection of surfactants for pulmonary delivery is very crucial part. Excipients approved by Food and Drug Administration (FDA) for pulmonary drug deliveries are limited and not accepted worldwide. Cationic and anionic surfactants showed toxicity and irritancy after pulmonary administration. Few nonionic surfactants are reported to be used; Poloxamer 188, Polysorbate, Lecithin for pulmonary drug delivery. $^{36}$

\section{Intravenous administration of SLNs}

Gastrointestinal tract (GIT) enzymatic degradation is acts as hurdle for oral administration of SLNs. The parenteral use of SLNs was extensively reviewed by. ${ }^{47}$

Aboutaleb et al., 2012 ${ }^{48}$ had formulated RIF loaded SLNs using modified microemulsion method. SLNs were characterized with respect to particle size, zeta potential, entrapment efficiency, surface morphology and in-vitro antibacterial activity. SLNs were found to be spherical with particle size $100 \mathrm{~nm}$ having low negative zeta potential. The minimum inhibitory concentration of RIF loaded SLNs was found to be eight times less than free RIF; thus antibacterial activity of RIF was successfully improved after encapsulation inside SLNs. The formulation showed sustained RIF release profile over the period of 72 hours. Thus authors concluded that; SLNs is promising avenue for enhanced antibacterial activity of RIF.

\section{SLNs based oral drug delivery for anti-tb chemotherapy}

Controlled drug release behavior of SLNs based system is reported to enable the prevention of gastric and intestinal degradation of the entrapped drug ${ }^{49,50}$ and their possible uptake and transport through intestinal mucosa. ${ }^{51}$ The adhesive properties of SLNs are reported to increases bioavailability and reduce erratic absorption.

Mucosa of intestine plays important role in absorption of drug by paracellular or intracellular uptake. However stability of SLNs in gastric fluid must be assess in order to predict their suitability for 
oral administration. Hydrophilic polymer coating is successful way to protect colloidal carrier from GIT enzymatic degradation.

Pandey et.al., $2005^{38}$ successfully entrapped anti-TB drugs (RIF, INH, PZY) in polyvinyl alcohol coated SLNs by emulsion solvent diffusion technique and following single oral administration to Laca mice, therapeutic drug concentration were maintained in the plasma for 8 days and in the organs (lungs, liver and spleen) for 10 days. Authors concluded that, SLNs can offer an economical and patient friendly approach for the administration of anti-TB drugs.

\section{CONCLUSION}

Although there are developed techniques of disease management, M.tb remains an important health problem worldwide. Lipid based nanoparticles distinguish themselves from other nanocarriers by many advantages, i.e., good tolerability, good stability, organic solvent free production and controlled drug release behavior. Review is therefore focused on potential use of SLNs for anti-TB chemotherapy. Among the various SLNs based drug delivery, direct delivery of drugs to the lungs seems appealing. However, there are also some requirements lipid nanoparticles have to fulfil for pulmonary administration. As alveolar macrophages acts as environment for survival and multiplication of M.tb active targeted delivery of drugs to macrophages is promising strategy. In addition, a low toxicological potential of pulmonary administered SLNs was demonstrated by several researchers. However, there is no investigation regarding long term toxicity study of lipid nanoparticles. $^{52}$

\section{REFERENCES}

1. Costa A, Pinheiro M, Magalhães J, et al. The formulation of nanomedicines for treating tuberculosis . Adv Drug Deliv Rev. 2016;102:102-115.

2. Sung JC, Pulliam BL, Edwards DA. Nanoparticles for drug delivery to the lungs. Trends Biotechnol. 2007;25(12):563-570.

3. Huang $\mathrm{C}$, Chiu P, Wang $\mathrm{Y}$, Chen $\mathrm{K}$. Electrochemically Controlling the Size of Gold Nanoparticles. J Electrochem Soc. 2006;153(12):193-198.

4. Lawlor C, Kelly C, Leary SO, et al. Cellular targeting and traf fi cking of drug delivery systems for the prevention and treatment of MTb. Tuberculosis. 2011;91(1):93-97.

5. Pham D, Fattal E, Tsapis N. Pulmonary drug delivery systems for tuberculosis treatment. Int J Pharm. 2015;478(2):517-529.

6. Couvreur P, Vauthier C. Expert Review Nanotechnology: Intelligent Design to Treat Complex Disease. Pharm Res. 2006;23(7):1417-1450.

7. Mehnert W, Mader K. Solid lipid nanoparticles Production, characterization and applications. Adv Drug Deliv Rev. 2001;47:165-196.

8. Russell DG, Barry CE, Flynn JL. Tuberculosis: What We Don' $\mathrm{t}$ Know. Tuberculosis Malaria. 2010;328:852-857.

9. Smith I. Mycobacterium tuberculosis Pathogenesis and Molecular Determinants of Virulence. Clin Microbiol Rev. 2003;16(3):463-496.

10. Zumla A, George A, Sharma V, et al. The WHO 2014 Global tuberculosis report further to go. Lancet Glob Heal. 2015;3(1):e10-e12.

11. Roach DR, Bean AGD, Demangel C, France MP, Briscoe H, Britton WJ. TNF Regulates 
Chemokine Induction Essential for Cell Recruitment, Granuloma Formation, and Clearance of Mycobacterial Infection.J Immunol. 2015;168:4620-4627.

12. Segovia-juarez JL, Ganguli S, ̃̃ DK. Identifying control mechanisms of granuloma formation during $\mathrm{M}$. tuberculosis infection using an agent-based model. J Theor Biol. 2004;231:357-376.

13. Kolyva AS, Karakousis PC. Old and New TB Drugs: Mechanisms of Action and Resistance, Understanding TuberculosisNew Approaches to Fighting Against Drug Resistance,ed. Cardona, D.P.-J. Baltimore,USA, 2009, p 209-232.

14. Lienhardt C, Vernon A, Raviglione MC. New drugs and new regimens for the treatment of tuberculosis : review of the drug development pipeline and implications for national programmes. Curr Opin. 2010;(Table 1):186193.

15. Swanson MS, Fernandez-moreia E, Swanson MS. A Microbial Strategy to Multiply in Macrophages : the Pregnant Pause. Traffic. 2002;3(3):170-177.

16. Alexandru-flaviu T, Cornel C. Macrophages Targeted Drug Delivery as a Key Therapy in Infectious Disease.Biotechnol Mol Biol Nanomedicine. 2014;2(1):19-21.

17. Vergne I, Fratti RA, Hill PJ, Chua J, Belisle J, Deretic V. Mycobacterium tuberculosis Phagosome Maturation Arrest : Mycobacterial Phosphatidylinositol Analog Phosphatidylinositol Mannoside Stimulates Early Endosomal Fusion. Mol Biol Cell. 2004; 15:751-760.

18. Dermine J, Desjardins M. Survival of intracellular pathogens within macrophages. Protoplasma. 1999:11-24.

19. Aderem A, Underhill DM. MECHANISMS OF PHAGOCYTOSIS IN MACROPHAGES. Annu Rev Immunol. 1999; 17:593-623.

20. Moghimi SM, Hunter AC. Recognition by Macrophages and Liver Cells of Opsonized Phospholipid Vesicles and Phospholipid Headgroups. Pharm Res. 2001;18(1):2-3.

21. Broekhoven CL Van, Parish CR, Demangel C, Britton WJ, Altin JG. Targeting Dendritic Cells with Antigen-Containing Liposomes: A Highly Effective Procedure for Induction of Antitumor Immunity and for Tumor Immunotherapy. Cancer Res. 2004;(24):4357-4365.

22. Takagi H, Numazaki M, Abe Y, Ishii M, Kato C, Kojima N. Cooperation of specific ICAM-3 grabbing nonintegrin-related 1 ( SIGNR1 ) and complement receptor type 3 ( CR3 ) in the uptake of oligomannose-coated liposomes by macrophages. Glycobiology. 2009;19(3):258-266.

23. Mcnally AK, Defife KM, Anderson JM. Interleukin-4-Induced Macrophage Fusion Is Prevented by Inhibitors of Mannose Receptor Activity. Am J Pathol. 1996;149(3):975-985.

24. $\tilde{A}$ UG, Martinez-pomares L. Influence of the mannose receptor in host immune responses. Immunobiology. 2009;214(7):554-561.

25. Bhardwaj A, Kumar L, Narang RK, Murthy RSR. Development and characterization of ligand-appended liposomes. Artif Cells, Nanomedicine, Biotechnol. 2013;142001(May 2012):52-59.

26. Lipoprotein M, Lrp RP, Krieger M, Herz J. 
Structure and functions of receptors: Macrophage Scavenger Receptors and LDL. Annu Rev Biochem. 1994;63:601-637.

27. Lee W, Loo C, Traini D, Young PM. Nanoand micro-based inhaled drug delivery systems for targeting alveolar macrophages.Expert Opin. 2015:1-18.

28. Gallagher JE, George G, Brody AR. Sialic Acid Mediates the Initial Binding of Positively Charged Inorganic Particles to Alveolar Macrophage Membranes 12. Am Rev Respir Dis. 1987;135(6):1345-1352.

29. Makino K, Yamamoto N, Higuchi K. Phagocytic uptake of polystyrene microspheres by al $\mathrm{v}$ eolar macrophages : effects of the size and surface properties of the microspheres. Colloids Surfaces B Biointerfaces. 2003;27:33-39.

30. Fontana G, Licciardi M, Mansueto S, Schillaci D. Amoxicillin-loaded polyethylcyanoacrylate nanoparticles: Infuence of PEG coating on the particle size , drug release rate and phagocytic uptake. Biomaterials. 2001;22:2857-2865.

31. Wretlind A. Development of Fat Emulsions. J Parenter Enter Nutr. 1980;5(3):230-235.

32. Schwarz C, Mehnert W, Lucks JS, Miiller RH. Solid lipid nanoparticles ( SLN ) for controlled drug delivery . I . Production, characterization and sterilization. J Control Release. 1994;30(93):83-96.

33. Sacks L V, Pendle S, Orlovic D, et al. Adjunctive Salvage Therapy with Inhaled Aminoglycosides for Patients with Persistent Smear-Positive Pulmonary Tuberculosis. Clin Infect Dis. 2001;32:44-49.

34. Condos R, Rom WN, Schluger
NW.Treatment of multidrug-resistant pulmonary tuberculosis with interferongamma via aerosol. Lancet Glob Heal. 1997;349(9064):1513-1515.

35. Weber S, Zimmer A, Pardeike J. Solid Lipid Nanoparticles ( SLN ) and Nanostructured Lipid Carriers ( NLC ) for pulmonary application: A review of the state of the art. Eur J Pharm Biopharm. 2013;86(1): 7-22

36. Pilcer G, Amighi K. Formulation strategy and use of excipients in pulmonary drug delivery.Int J Pharm. 2010;392(1-2):1-19.

37. Li H. The use of amino acids to enhance the aerosolisation of spray-dried powders for pulmonary gene therapy. J Gene Med. 2005;7:343-353.

38. Pandey R, Sharma S, Khuller GKÃ. Oral solid lipid nanoparticle-based antitubercular chemotherapy. Tuberculosis. 2005;85:415420.

39. Chuan J, Li Y, Yang L. Enhanced rifampicin delivery to alveolar macrophages by solid lipid nanoparticles. J Nanoparticle Res. 2013;15(5):1634.

40. Vieira ACC, Chaves LL, et al. Mannosylated solid lipid nanoparticles for the selective delivery of rifampicin to macrophages. Artif Cells, Nanomedicine, Biotechnol. 2018;46:653-663.

41. Maretti E, Rossi T, Bondi M, et al. Inhaled Solid Lipid Microparticles to target alveolar macrophages for tuberculosis. Int J Pharm. 2014;462(1-2):74-82.

42. Blasi P, Giovagnoli S, Schoubben A, Ricci M, Rossi C. Solid lipid nanoparticles for targeted brain drug delivery. Adv Drug Deliv Rev. 2007;59:454-477. 
43. Cavalli R, Caputo O, Eugenia M, Trotta M, Scarnecchia C, Gasco MR. journal of pharmaceutics Sterilization and freeze-drying of drug-free and drug-loaded solid lipid nanoparticles. Int J Pharm. 1997;148:47-54.

44. Heiati H, Tawashi R, Phillips NC. Drug retention and stability of solid lipid nanoparticles containing azidothymidine palmitate after autoclaving, storage and lyophilization.J Microencapsul. 2008;15(2):173-184.

45. Nayak AP, Tiyaboonchai W, Patankar S, Madhusudhan B, Souto EB. Colloids and Surfaces B: Biointerfaces Curcuminoidsloaded lipid nanoparticles: Novel approach towards malaria treatment.Colloids Surfaces B Biointerfaces. 2010;81(1):263-273.

46. Pardeike J, Weber $\mathrm{S}$, Haber $\mathrm{T}$, et al. Development of an Itraconazole-loaded nanostructured lipid carrier ( NLC ) formulation for pulmonary application. Int $\mathrm{J}$ Pharm. 2011;419(1-2):329-338.

47. Wissing SA, Kayser O, Mu RH. Solid lipid nanoparticles for parenteral drug delivery. Adv Drug Deliv Rev. 2004;56:1257-1272.

48. Aboutaleb E, Noori M, Gandomi N, Atyabi F, Fazeli MR. Improved antimycobacterial activity of rifampin using solid lipid nanoparticles. Int Nano Lett. 2012;2(1): 1-8.

49. Damge C, Michel C,Aprahamian M, Couvreu P, Devissaguet J. Nanocapsules as carrier for oral peptide delivery. J Control Release. 1990;13:233-239.

50. Olbrich $\mathrm{C}$, Kayser $\mathrm{O}, \mathrm{Mu}$ RH. Lipase degradation of Dynasan 114 and 116 solid lipid nanoparticles ( SLN ) effect of surfactants, storage time and crystallinity. Int
J Pharm. 2002;237:119-128.

51. Tob1 M, Sa A. The role of PEG on the stability in digestive fluids and in vivo fate of PEG-PLA nanoparticles following oral administration. Colloids Surfaces B Biointerfaces. 2000;18:315-323.

52. Hyeon T, Rotello V. Nanomedicine themed issue. Chem Soc Rev. 2012;41(7):2971-3010.

53. Liu J, Gong $\mathrm{T}, \mathrm{Fu} \mathrm{H}$, et al. Solid lipid nanoparticles for pulmonary delivery of insulin. Int J Pharm. 2008;356:333-344.

54. Dharmala K, Yoo JW, Lee CH. Development of Chitosan - SLN Microparticles for chemotherapy: In vitro approach through ef fl ux-transporter modulation. J Control Release. 2008;131(3):190-197.

55. Chattopadhyay P, Shekunov BY, Yim D, Cipolla D, Boyd B, Farr S. Production of solid lipid nanoparticle suspensions using supercritical fluid extraction of emulsions ( SFEE ) for pulmonary delivery using the AERx system . Adv Drug Deliv Rev. 2007;59:444-453.

56. Esmaeili M, Aghajani M, Abbasalipourkabir R. Budesonide-loaded solid lipid nanoparticles for pulmonary delivery: preparation, optimization, and aerodynamic behavior. Artif Cells, Nanomedicine, Biotechnol. 2016; 44(8)1964-1971.

57. Li Y, Sun X, Gong T, Liu J, Zuo J, Zhang Z. Inhalable Microparticles as Carriers for Pulmonary Delivery of Thymopentin-Loaded Solid Lipid Nanoparticles. Pharm Res. 2010;27:1977-1986.

58. Patlolla RR, Chougule M, Patel AR, Jackson T, Tata PN V, Singh M. Formulation, characterization and pulmonary deposition of 
nebulized celecoxib encapsulated nanostructured lipid carriers. J Control Release. 2010;144(2):233-241.

59. Rudolph C, Schillinger U, Ortiz A, et al. Application of Novel Solid Lipid Nanoparticle ( SLN ) -Gene Vector Formulations Based on a Dimeric HIV-1
TAT-Peptide in Vitro and in Vivo.Pharm Res. 2004;21(9):1662-1669.

60. Videira M, Almeida AJ, Fabra À. Preclinical evaluation of a pulmonary delivered paclitaxel-loaded lipid nanocarrier antitumor effect. Nanomedicine Nanotechnology, BiolMed. 2012;8(7):1208-1215. 